\title{
Rodents for comparative aging studies: from mice to beavers
}

\author{
Vera Gorbunova • Michael J. Bozzella • \\ Andrei Seluanov
}

Received: 2 April 2008 /Accepted: 14 April 2008 /Published online: 25 June 2008

(C) American Aging Association 2008

\begin{abstract}
After humans, mice are the best-studied mammalian species in terms of their biology and genetics. Gerontological research has used mice and rats extensively to generate short- and long-lived mutants, study caloric restriction and more. Mice and rats are valuable model organisms thanks to their small size, short lifespans and fast reproduction. However, when the goal is to further extend the already long human lifespan, studying fast aging species may not provide all the answers. Remarkably, in addition to the fast-aging species, the order Rodentia contains multiple long-lived species with lifespans exceeding 20 years (naked mole-rat, beavers, porcupines, and some squirrels). This diversity opens great opportunities for comparative aging studies. Here we discuss the evolution of lifespan in rodents, review the biology of slow-aging rodents, and show an example of how the use of a comparative approach revealed that telomerase activity coevolved with body mass in rodents.
\end{abstract}

Keywords Aging - Comparative studies .

Rodents $\cdot$ Telomerase

The focus of this review is on the use of long-lived rodent species for comparative aging studies. We

V. Gorbunova $(\bowtie) \cdot$ M. J. Bozzella $\cdot$ A. Seluanov

Department of Biology, University of Rochester,

213 Hutchison Hall,

Rochester, NY 14627, USA

e-mail: vera.gorbunova@rochester.edu discuss the evolution of lifespan in rodents, and show that long-lifespan evolves when extrinsic mortality is low. We review the biology of several slow-aging rodent species and their potential use as models for biogerontological research. Finally, we discuss our recent study, which used a comparative approach to reveal that telomerase activity coevolved with body mass in rodents.

Rodents are among the most successful mammals. Over $40 \%$ of all mammalian species belong to order Rodentia. In other words, slightly less than half of all mammals are rodents. Moreover, these animals are very numerous, are found worldwide, and are absent only in Antarctica, New Zealand and some islands (Nowak 1999; Macdonald 2001). Some rodent species have shown an undesirable devotion to humans, and have influenced human history as food pests and carriers of fatal diseases. More recently, rodents became important laboratory animals, and a great deal of progress in biomedical research can be attributed to the use of these formerly obnoxious animals. Mice and rats are small, fast reproducing, and easy to maintain in captivity. They are also very short lived, with the longest recorded lifespans being 4 and 5 years for mice and rats, respectively. All these qualities make mice and rats ideal laboratory animals. In gerontological research, the first mammalian mutants with altered lifespan were generated in mice (reviewed in Hasty et al. 2003; Bartke and BrownBorg 2004; Hasty and Vijg 2004), and mice and rats were the first mammals to reveal the life-prolonging 
effect of caloric restriction (Masoro 1992). While the short lifespan of mice and rats may seem beneficial for a laboratory animal, there is an important limitation. Since the goal of aging research is to achieve life extension in humans - one of the slowest aging mammals - studying the fastest aging species may not provide all the desired clues. Remarkably, order Rodentia includes species with highly diverse aging rates. Many species, such as naked mole-rat (Heterocephalus glaber), beavers (Castor canadensis and Castor fiber), porcupines (Erethizon dorsatum, Coendou prehensilis, Petaurista alborufus, Atherurus africanus, Hystrix indica, Hystrix brachyura, Hystrix africaeaustralis, and Hystrix cristata), and several squirrels (Sciurus carolinensis, Callosciurus prevostii, Ratufa indica and Petaurista alborufus) have documented lifespans of over 20 years (Buffenstein and Jarvis 2002; Austad 2005; Weigl 2005). This diversity can be exploited to understand mechanisms of aging.

Long-lived rodent species may be particularly useful for aging studies given their relationship to standard laboratory aging models-mice and rats. Historically, the comparative approach has provided spectacular advances in biology, the best example being Darwin's discovery of the principle of natural selection. Later the focus has shifted to the studies of a few model species, which yielded significant discoveries in cell and molecular biology. Today, with the new molecular tools available to study agingrelated cellular and physiological mechanisms, the comparative approach can be utilized at a qualitatively new level. A dramatic step toward understanding the molecular mechanisms of human aging would be achieved if we could discover the mechanisms responsible for the more than 10-fold difference in lifespan between rodent species.

\section{Rodent systematics}

Despite numerous morphological and molecular studies, the higher-order systematics of rodents is a subject of intense debate. The difficulties arise because similar morphological traits used for rodent classification have evolved independently in multiple clades, the sparse fossil record, and also the high rate of nucleotide substitutions observed in DNA studies. Traditionally, the order Rodentia is divided into two suborders: Sciurognathi and Hystricognathi (Fig. 1)

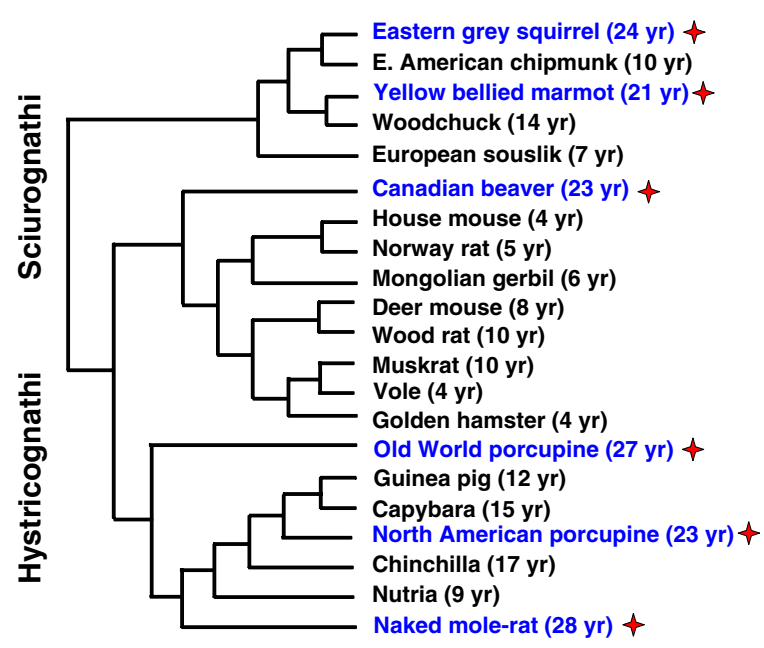

Fig. 1 Rodent phylogeny. The tree topology is based on molecular phylogenies inferred from Martin et al. 2000; Michaux et al. 2001; Murphy et al. 2001; Montgelard et al. 2002; Adkins et al. 2003; and Steppan et al. 2004). Stars indicate species with lifespan longer than 20 years

(Martin et al. 2000; Michaux et al. 2001; Murphy et al. 2001; Montgelard et al. 2002; Adkins et al. 2003; Steppan et al. 2004) Squirognathi are divided into two infraorders: Squirrel-like rodents and Mouse-like rodents.

Squirrel-like rodents include seven families: squirrels (Sciuridae), beavers (Castoridae); Aplodontidae with a single species, the mountain beaver; pocket gophers (Geomyidae); Scaly-tailed squirrels (Anomaluridae); pocket mice and kangaroo mice or rats (Heteromyidae); and Pedetidae with one species, the jumping "hare".

Mouse-like rodents include five families. The main family is Muridae, which contains the greatest number of species (approximately 1,082). The subfamilies within Muridae are: Old World mice and rats (Murinae); New World mice and rats (Sigmodontiae); voles (Arvicolinae); and hamsters (Cricetinae). Other families are dormice (Gliridae and Seleviniidae), jumping mice (Zapodidae), and jerboas (Dipodidae).

Histricognathi, or cavy-like rodents include species living in Central and South America, but some are African. The suborder includes 18 families, among them New World porcupines (Erethizontidae), cavies (Caviidae, includes well-known guinea pig), Hydrochoeridae with a single species, the capybara (Hydrochoerus hydrochoeris). The capybara is the largest rodent, reaching 65,000 $\mathrm{g}$ in body weight (Macdonald 2001). Some other families are nutrias (Myocastor- 
idae), pacas (Agoutidae), chinchillas (Chinchillidae), and five, mainly African, families of African and Asian porcupines (Hystricidae), spiny rats (Thryonomyidae), rock mice or rats (Petromyidae), gundis (Ctenodactylidae), and African mole-rats (Bathyeridae).

\section{Evolution of lifespan in rodents}

Unless otherwise specified, data on maximum lifespans for the wild rodent species discussed below were obtained from the monograph by Richard Weigl, Longevity of Mammals in Captivity; from the Living Collections of the world (Weigl 2005), and are based on zoo records for which both the birth and the death dates of the animals were documented. In comparison with the lifespan data of laboratory mice, the maximum lifespans of wild species can be considered underestimates of their physiological lifespan potential, due to the smaller size of the studied populations.

Rodents are phylogenetically related, yet their lifespans are extremely diverse. In many cases, species within the same family show large differences in lifespan. Within the single family Sciuridae, lifespans differ by more than two-fold between tree squirrels and chipmunks. The eastern grey squirrel (Sciurus carolinensis) has a maximum lifespan of 24 years. Similarly long lifespans were documented for several other species of tree squirrels and for flying squirrels: Prevost's squirrel (Callosciurus prevostii) has a lifespan of 21 years; the Indian giant squirrel (Ratufa indica) lives for 20 years; and the red and white flying squirrel (Petaurista alborufus) lives as long as 21 years in captivity. Relatives of tree squirrels, chipmunks, which spend their life on the ground, live considerably shorter lives: the Eastern American chipmunk (Tamias striatus) has a maximum lifespan of 9.5 years. A similar lifespan has been documented for the Siberian chipmunk (Tamias sibiricus) and several other chipmunks. Ground squirrels are also shorter-lived: the black-tailed prairie dog (Cynomys ludovicianus) has a maximum lifespan of 11 years; and the European souslik (Spermophilus citellus) as well as several other sousliks live for under 7 years. Larger marmots include species with long lifespans such as 21 years for the yellow-bellied marmot (Marmota flaviventris), to shorter-lived species like the common woodchuck (Marmota monax), which has been documented to reach an age of only 14 .
Family Muridae contains primarily short-lived species, although there is considerable variability here as well. Old world mice, rats, and hamsters have maximum lifespans of under 5 years. Similar New World species such as deer mouse (Peromyscus maniculatus) or white-footed mouse (Peromyscus leucopus) live up to 8 years, and wood rat (Neotoma albigula), and desert wood rat (Neotoma lepida) live up to 10 years. New world mice and rats are very similar to Old world mice and rats in body size and ecology, thus the almost twofold difference in maximum lifespan is very intriguing, and has been investigated in comparative studies (Sohal et al. 1993; Csiszar et al. 2007).

The family Castoridae has only two species: Canadian beaver and Eurasian beaver. The longevity record for Canadian beavers is 23 years. There is not enough data for the Eurasian beavers for an accurate estimation of lifespan but various sources suggest that this beaver is also very long-lived. There are claims that it has reached an age of 35-50 years in zoos (Marcuzzi 1986).

The suborder Histricognathi includes both longlived and relatively short-lived species (Weigl 2005). The maximum lifespan of the domestic guinea pig (Cavia porcellus) is 12 years. Its wild relatives, the Brazilian guinea pig (Cavia aperea) and Peruvian guinea pig (Cavia tschudii), have been reported to reach an age of only 6 years. The world's largest rodent, the capybara, has a maximum lifespan of 15 years, which seems rather short considering its large size (up to $65 \mathrm{~kg}$ body mass). Another cavy-like rodent, the Chinchilla (Chinchilla lanigera), has a longer lifespan. The documented record for a chinchilla is 17 years, but chinchilla breeders claim that some of their pets have lived over 20 years. The chinchilla is similar in size and ecology to the guinea pig, but lives in barren regions at higher elevations, and may have fewer predators in the wild.

The two families of porcupines, New World porcupines and Old World porcupines are only distantly related, but both are extremely long-lived (Weigl 2005). The Old world porcupine (Hystrix brachyura), African porcupine (Hystrix cristata), and Indian crested porcupine (Hystrix indica) have maximum lifespans of 27-28 years. The North American porcupine (Erethizon dorsatum) has been documented to live 23 years, but its South American relative Prehensile-tailed porcupine (Coendou prehensilis) has lived for 27 years. 
The most extreme longevity relative to body size is observed in the naked mole-rat (Heterocephalus glaber). About the size of a mouse, naked mole rats have lived as long as 28 years in captivity (Buffenstein and Jarvis 2002). Mole-rats are African rodents that spend most of their life in underground burrows. The family includes other long-lived species such as Ansell's mole-rat (Cryptomys anselli), with maximum lifespan of 21 years, and the shorter-lived Cape molerat (Georychus capensis), with the longest recorded lifespan of 11 years (Weigl 2005). Naked mole-rats and common mole-rats (genus Cryptomys) are social animals living in large colonies, while Cape mole-rats are solitary. Mole rats are far less exposed to predators than are surface-dwelling rodents. The only threat comes from snakes that can penetrate their deep burrows. Social mole-rats use cooperative defense against snakes, which may further reduce their mortality relative to solitary mole-rats.

There are drastic differences among rodent species in the level of predation to which they are subject. Short-lived muroid rodents are subject to heavy predation, while long-lived rodents such as porcupine, beaver, or naked mole-rat have few or no predators. Long-lived rodents are protected from predators through various means. Tree squirrels and flying squirrels live high above the ground. Porcupines are large, and are covered with quills. Beavers are also large and spend most of their life in or near the water in cleverly constructed protective lodges. Naked mole-rats live in deep underground burrows and collectively defend against snakes. Longer lifespans in rodent species with special defenses are consistent with the classical theory for evolution of senescence, which states that long lifespans evolve when extrinsic mortality is low (Medawar 1952; Williams 1957; Kirkwood and Austad 2000). Extremely long-lived rodent species occur in several distantly related phylogenetic groups (Fig. 1), including mole-rats, porcupines, beavers, and squirrels, indicating that slow aging has independently evolved multiple times in rodents (Austad 2005). This makes rodents especially valuable for aging research because it may allow identification of molecular and physiological traits that co-evolve with slow aging in convergent species.

Rodents also differ dramatically in their body mass. Among the smallest rodent species, mice have an average body mass of $10-30 \mathrm{~g}$, while the largest rodent, the capybara, weighs up to $65,000 \mathrm{~g}$ (Nowak 1999). This diversity is very useful for comparative aging studies since many traits, such as lifespan, metabolic rate, cellular senescence, and telomerase activity show dependence on body mass (Austad and Fischer 1991; Lorenzini et al. 2005; Seluanov et al. 2007). Rodents include species with all possible combinations of lifespan and body mass: species with large body mass and average lifespan (capybara), large body mass and long lifespan (beaver and porcupine), small body mass and short lifespan (mouse), and small body mass and long lifespan (mole-rats).

\section{Biology of some long-lived species}

The following sections discuss the four longest-lived rodents: Eastern grey squirrel, American beaver, North American porcupine, and naked mole-rat. All four species have documented lifespans exceeding 23 years, and are especially interesting for comparative aging studies. We discuss the biology of these species, adaptations that have contributed to evolution of their long lifespan, and the potential use of these animals for aging research.

\section{Eastern grey squirrel}

The Eastern grey squirrel is a beautiful animal that adapted to living alongside humans in city parks and backyards, often becoming a pest. Most people not familiar with animals of this species are surprised by their exceptional longevity. The Eastern grey squirrel is a medium-sized rodent with an average body mass of $500 \mathrm{~g}$ (Grzimek 1990; Nowak 1999; Macdonald 2001). This species is represented everywhere in the Eastern United States. It is strictly diurnal with excellent eyesight. The Eastern grey squirrel consumes a variety of foods, including insects, nestling birds, fruits, various seeds, and nuts. The squirrel is primarily a climbing, tree animal that visits the ground only for gathering food and for burying or digging out nuts. The most important predators of Eastern Gray Squirrels are hawks, owls, foxes, raccoons, and snakes. Squirrels are, however, a difficult prey to catch due to their excellent tree climbing and jumping ability. Eastern grey squirrels produce one litter in the spring, although older 
females may occasionally produce a second litter in the fall. One female mates with several males after leading them on a spectacular chase. The litter size is usually $2-5$. The young become independent by 10 weeks of age, and reach sexual maturity at about 1 year. Eastern grey squirrels do not hibernate, are adaptable, and are easy to maintain in captivity. This makes it a promising long-lived model organism for biogerontologists, as an alternative to fast-aging mice and rats.

\section{North American beaver}

After the capybaras, beavers are the largest rodents in the world; adults can weigh up to 45,000 g (Grzimek 1990; Nowak 1999; Macdonald 2001). Beavers are semi-aquatic and always live near the water. Beavers display complex construction, communication, and social behavior that set them apart from other mammals. Like humans, beavers build homes (lodges and burrows), and modify the environment by building dams and canals. Furthermore, beavers are unusual among rodents in that they form monogamous pairs. The mated pair stays together until one adult dies. Beavers live in small family units consisting of an adult pair, young born in the current year, and older subadults from previous seasons. The average litter size is 1-3 kits, and the young stay with the family for 2 years. Beavers are herbivores, feeding on leaves, grasses, roots, shrubs, and trees. Beaver predators are primarily humans, bears, lynx, wolves, and wolverines. Beavers, however, are very strong and are able to drown a dog or wolf by pulling them under the water. The beavers cover their lodges in late autumn with fresh mud, which freezes when the frost sets in. The mud becomes almost as hard as stone, so that predators cannot get in. Due to its large size and complex housing needs, the beaver is an unlikely laboratory animal. However, cell and tissue specimens obtained from wild beavers may be useful in biogerontological studies.

\section{North American Porcupine}

The North American porcupine is found from Mexico to Alaska and is a very widely known, large nocturnal rodents (up to 9,000 g body weight) (Grzimek 1990; Nowak 1999; Macdonald 2001). Porcupines are protected from predators by sharp quills, which represent modified hairs. The quills detach easily and penetrate the predator's skin. The quills also have microscopic scales that point backwards that make them hard to remove. The quills are normally flattened against the body unless the animal is disturbed. Porcupines also defend themselves by swinging their quilled tail towards a perceived threat. Porcupines are slow moving, and did not evolve to be fast since running away is not their defense strategy. Nevertheless, they are excellent climbers, and spend time on trees feeding on seeds, leaves, fruit, and flowers. Porcupines do not hibernate, and, in the winter, feed on tree bark and conifer needles. The North American porcupine is solitary. Females become reproductively mature at 18 months of age, and usually produce a single, large newborn with soft quills that harden within a few hours after birth. Similar to beavers, porcupines are too large and difficult to handle to become a laboratory animal, but samples obtained from wild animals may prove valuable for aging studies.

\section{Naked mole-rat}

The naked mole-rat may not be as aesthetically pleasing as a squirrel, but it is nevertheless one of the most interesting rodent species. The naked molerat is the only eusocial mammal living in large colonies with one breeding female, the queen (Grzimek 1990; Nowak 1999; Macdonald 2001; Buffenstein 2005). The queen is the dominant individual in the colony. Other females are capable of becoming breeders, but their estrous cycles are suppressed in the presence of the queen. The offspring are cared for by the breeding female, as well as by other members of the colony. Naked molerats live strictly underground, feeding on roots and tubers. The naked mole rat is a small rodent with average body mass of $35 \mathrm{~g}$. Interestingly, this species has almost lost the ability to regulate body temperature, as in their natural habitat the underground temperature is constant $28-30^{\circ} \mathrm{C}$. Their metabolic rate is approximately half that predicted allometrically. Naked mole-rat colonies can be successfully maintained in captivity and are becoming a popular model organism for aging research (Buffenstein 2005). The detailed review of naked mole-rat physiology is provided in [see Buffenstein et al. 2008 (this issue)]. 


\section{Coevolution of telomerase repression and body mass in rodents}

We used the diversity of rodent species to resolve the long-standing paradox concerning the evolution of telomerase regulation. Telomerase is a specialized cellular reverse transcriptase responsible for maintaining telomere length. In the absence of telomerase, linear chromosomes cannot be fully replicated and shorten with every cell division (the so-called end replication problem) (Olovnikov 1973). In human somatic cells telomerase is not expressed (Harley et al. 1990). As a result, when telomeres of human somatic cells reach a critically short length the cells enter irreversible growth arrest called replicative senescence (Shay and Wright 2000). Replicative senescence is believed to be an anti-cancer mechanism that limits cell proliferation (Campisi 2001).

The house mouse, despite being a staple mammal for biomedical research, differs dramatically from human in terms of its telomere biology. Adult mice express telomerase in multiple somatic tissues (Prowse and Greider 1995). Moreover, telomeres of mouse cells do not shorten with cell divisions, and mouse fibroblasts do not exhibit replicative senescence when cultured under physiological oxygen concentration (Parrinello et al. 2003). The strikingly different telomere biology of humans and mice has been explained by their differences in lifespan and body mass (Wright and Shay 2000; Forsyth et al. 2002). Mice are short-lived, dying mostly of predation in the wild; thus they do not need efficient anticancer mechanisms. Other evidence for the lack of efficient anticancer defenses in mice includes the observation that $90 \%$ of mice in captivity die of cancer (Lipman et al. 2004). In contrast, as long-lived organisms, it has been suggested that humans have evolved genetic systems that strictly limit cell proliferation (Wright and Shay 2000). Similarly, telomerase activity may coevolve with body mass. Humans are larger than mice, and their bodies contain many more cells. Since malignant transformation may occur in any single cell, humans likely require more efficient anticancer mechanisms to mitigate the cancer risk conferred by their large size.

To test whether telomerase activity coevolves with lifespan or body mass, we examined telomerase activity in large and/or long-lived rodent species. We analyzed telomere biology in a collection of 15 rodent species with diverse lifespans and body masses (Seluanov et al. 2007). Telomerase activity was measured in seven tissues of multiple individuals of each species using telomeric repeat amplification protocol, and a combined value "telomerase activity coefficient" was calculated for each species. Most rodents showed high telomerase activity in multiple somatic tissues. Surprisingly, high telomerase activity was found in the longest living rodents: the naked mole-rat and the Eastern grey squirrel. The two species almost completely lacking telomerase activity were the beaver and the capybara, which are the largest rodents.

We then statistically evaluated the relationship between telomerase activity, body mass, and lifespan. The analysis revealed significant negative correlation between telomerase activity and body mass $\left(F_{1,13}=\right.$ $\left.14.9, r^{2}=0.534, P=0.002\right)$, but no correlation between telomerase activity and lifespan $\left(F_{1,13}=1.2, r^{2}=0.085\right.$,
Fig. 2 Correlation of telomerase activity with (a) body mass, and (b) maximum lifespan
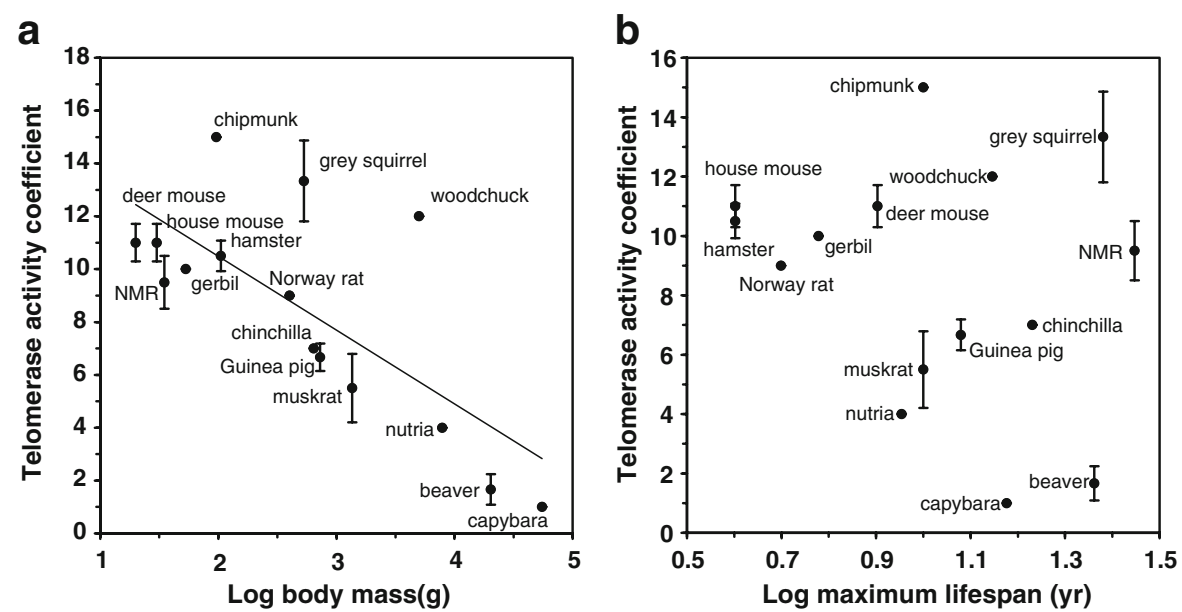
$P=0.293$ ) (Fig. 2). Namely, larger species have stronger repression of somatic telomerase activity.

In order to draw conclusions concerning coevolution of traits, it is essential to correct the species data for possible effects of shared phylogenetic ancestry (Felsenstein 1985). Ignoring phylogeny may lead to mistaken inferences. For example, a study analyzing coevolution of physical traits and lifespan and using five species of apes and five species of mice, might mistakenly infer that long lifespan coevolves with the lack of a tail. Since the analysis involves ten species the correlation may appear significant. However, considering that all apes have a common ancestor, just as all mice have a common ancestor, the true sample size in this comparison is 1 . In other words, all apes are long lived and lack tails, likely because their common ancestor was long-lived and lost a tail-not because each species has independently evolved long lifespan and the lack of a tail. The importance of considering phylogenies in comparative biology was recognized more than 20 years ago (Felsenstein 1985), and multiple software packages have been developed to transform raw species data into phylogenetically independent contrasts.

In our study (Seluanov et al. 2007), we generated independent contrasts for phenotypic values using the Comparative Analysis by Independent Contrasts (CAIC) program (Purvis and Rambaut 1995). The correlation between telomerase activity and body mass remained significant following phylogenetic correction $\left(F_{1,13}=42.1, r^{2}=0.76, P<0.0001\right)$, and also after correction for possible correlation between body mass and lifespan (Seluanov et al. 2007). Thus, reduced telomerase activity appears to have evolved in larger but not in longer-lived species (Seluanov et al. 2007).

In order to explain the correlation between telomerase activity and body mass (Fig. 3), we propose the following model: when evolution results in an increased body mass for a species, it follows that the species also exhibits an increased risk of developing cancer. Larger bodies contain more cells, each of which may potentially become cancerous. For example, an average beaver's body mass is approximately 2,000 times that of a mouse; it therefore may be expected that the average beaver will be 2,000 times more likely to develop a malignancy. Unfortunately, there is little data available on the cancer rates in beavers or other large rodents; even so, when one

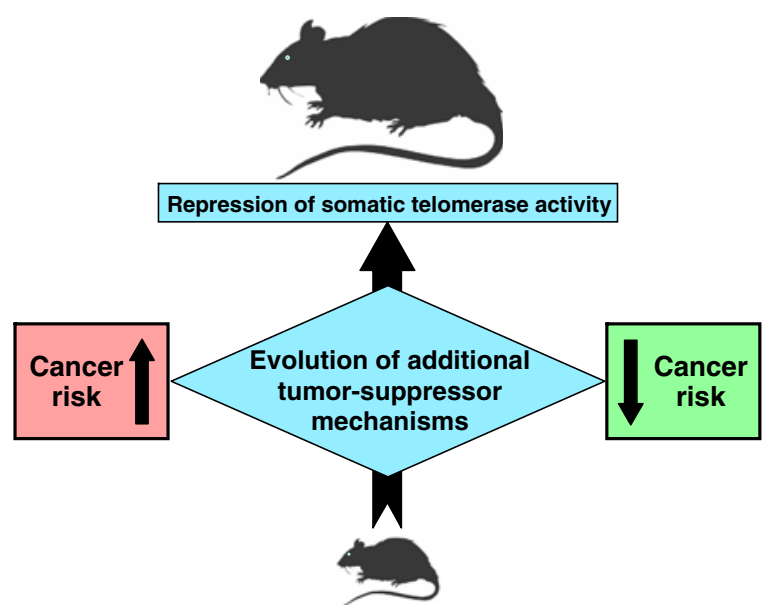

Fig. 3 A model explaining coevolution of telomerase activity and body mass. Evolutionary increases in body mass lead to increased cancer risk. To counteract this risk, large species evolve additional tumor-suppressor mechanisms such as the repression of telomerase activity in somatic cells

combines the fact that larger rodents live considerably longer than mice, and that their species continue to thrive, it can be suggested that they have evolved efficient tumor-suppressor mechanisms to mitigate the increased risk of cancer. We propose the repression of telomerase in somatic tissues as adaptive tumorsuppressor mechanism that evolves with increased body mass (Seluanov et al. 2007).

The notion that cancer may impose a selection that drives the adaptive evolution of tumor-suppressor mechanisms has been proposed previously (Graham 1983; Nunney 1999; Leroi et al. 2003). The prediction was that the larger an animal, the greater the number of tumor-suppressor genes it would possess, and that it would require more deleterious events for tumor formation. The theory gained its first experimental support with the data obtained on rodents. Nevertheless, there may be other tumor-suppressor mechanisms that evolve with body mass beyond telomerase suppression (Promislow 1994).

Another factor that would be thought to increase life-time cancer risk is a longer lifespan. For that reason, it is intriguing that we found no correlation between telomerase activity and lifespan (Seluanov et al. 2007). We can offer two possibilities as to why this is the case. First, cancer risk stemming from body mass may be greater than the risk resulting from lifespan; second, there may be unique risk factors associated with each particular set of anti-tumor 
adaptations. While a more massive body drives the evolution of replicative senescence, greater longevity may be related to separate adaptations, such as more precise DNA repair.

The study of telomerase in rodents (Seluanov et al. 2007) demonstrates how a comparative approach can help solve biological problems. Furthermore, the diversity of lifespans and body sizes displayed by the rodent species may greatly benefit biogerontology.

Acknowledgments Work in the authors' laboratory is supported by grants from US National Institute of Aging, Ellison Medical Foundation, and Susan Komen Foundation to V.G., and Ellison Medical Foundation to A.S.

\section{References}

Adkins RM, Walton AH, Honeycutt RL (2003) Higher-level systematics of rodents and divergence time estimates based on two congruent nuclear genes. Mol Phylogenet Evol 26:409-420

Austad SN (2005) Diverse aging rates in metazoans: targets for functional genomics. Mech Ageing Dev 126:43-49

Austad SN, Fischer KE (1991) Mammalian aging, metabolism, and ecology: evidence from the bats and marsupials. $\mathrm{J}$ Gerontol 46:B47-B53

Bartke A, Brown-Borg H (2004) Life extension in the dwarf mouse. Curr Top Dev Biol 63:189-225

Buffenstein R (2005) The naked mole-rat: a new long-living model for human aging research. J Gerontol A Biol Sci Med Sci 60:1369-1377

Buffenstein R, Jarvis JU (2002) The naked mole rat - a new record for the oldest living rodent. Sci Aging Knowledge Environ 2002:pe7

Buffenstein R, Edrey YH, Yang T, Mele J (2008) The oxidative stress theory of aging: embattled or invincible? Insights from non-traditional model organsims. AGE doi:10.1007/ s11357-008-9058-z

Campisi J (2001) Cellular senescence as a tumor-suppressor mechanism. Trends Cell Biol 11:S27-S31

Csiszar A, Labinskyy N, Zhao X, Hu F, Serpillon S, Huang Z, Ballabh P, Levy RJ, Hintze TH, Wolin MS, Austad SN, Podlutsky A, Ungvari Z (2007) Vascular superoxide and hydrogen peroxide production and oxidative stress resistance in two closely related rodent species with disparate longevity. Aging Cell 6:783-797

Felsenstein J (1985) Phylogenies and the comparative method. Am Nat 125:1-15

Forsyth NR, Wright WE, Shay JW (2002) Telomerase and differentiation in multicellular organisms: turn it off, turn it on, and turn it off again. Differentiation 69:188-197

Graham J (1983) Cancer and evolution: synthesis. J Theor Biol 101:657-659

Grzimek B (1990) Grzimek's animal life encyclopedia. McGraw-Hill, New York
Harley CB, Futcher AB, Greider CW (1990) Telomeres shorten during ageing of human fibroblasts. Nature 345:458-460

Hasty P, Vijg J (2004) Accelerating aging by mouse reverse genetics: a rational approach to understanding longevity. Aging Cell 3:55-65

Hasty P, Campisi J, Hoeijmakers J, van Steeg H, Vijg J (2003) Aging and genome maintenance: lessons from the mouse. Science 299:1355-1359

Kirkwood TB, Austad SN (2000) Why do we age. Nature 408:233-238

Leroi AM, Koufopanou V, Burt A (2003) Cancer selection. Nat Rev Cancer 3:226-231

Lipman R, Galecki A, Burke DT, Miller RA (2004) Genetic loci that influence cause of death in a heterogeneous mouse stock. J Gerontol A Biol Sci Med Sci 59:977-983

Lorenzini A, Tresini M, Austad SN, Cristofalo VJ (2005) Cellular replicative capacity correlates primarily with species body mass not longevity. Mech Ageing Dev 126:1130-1133

Macdonald D (ed) (2001) The new encyclopedia of mammals. Oxford University Press, Oxford

Marcuzzi G (1986) Man-beaver relations. In: Pilleri G (ed) Investigations on Beavers. Brain Anatomy Institute, Berne, pp 16-72

Martin Y, Gerlach G, Schlotterer C, Meyer A (2000) Molecular phylogeny of European muroid rodents based on complete cytochrome b sequences. Mol Phylogenet Evol 16:37-47

Masoro EJ (1992) The role of animal models in meeting the gerontologic challenge of the 21 st century. Gerontologist 32:627-633

Medawar PB (1952) An unsolved problem of biology. Lewis, London

Michaux J, Reyes A, Catzeflis F (2001) Evolutionary history of the most speciose mammals: molecular phylogeny of muroid rodents. Mol Biol Evol 18:2017-2031

Montgelard C, Bentz S, Tirard C, Verneau O, Catzeflis FM (2002) Molecular systematics of sciurognathi (rodentia): the mitochondrial cytochrome $b$ and 12S rRNA genes support the Anomaluroidea (Pedetidae and Anomaluridae). Mol Phylogenet Evol 22:220-233

Murphy WJ, Eizirik E, Johnson WE, Zhang YP, Ryder OA, O'Brien SJ (2001) Molecular phylogenetics and the origins of placental mammals. Nature 409:614-618

Nowak R (1999) Walker's mammals of the world. John Hopkins University Press, Baltimore

Nunney L (1999) Lineage selection and the evolution of multistage carcinogenesis. Proc Biol Sci 266:493-498

Olovnikov AM (1973) A theory of marginotomy. The incomplete copying of template margin in enzymic synthesis of polynucleotides and biological significance of the phenomenon. J Theor Biol 41:181-190

Parrinello S, Samper E, Krtolica A, Goldstein J, Melov S, Campisi J (2003) Oxygen sensitivity severely limits the replicative lifespan of murine fibroblasts. Nat Cell Biol 5:741-747

Promislow DE (1994) DNA repair and the evolution of longevity: a critical analysis. J Theor Biol 170:291-300

Prowse KR, Greider CW (1995) Developmental and tissuespecific regulation of mouse telomerase and telomere length. Proc Natl Acad Sci USA 92:4818-4822

Purvis A, Rambaut A (1995) Comparative analysis by independent contrast (CAIC): an Apple Macintosh appli- 
cation for analysing comparative data. Comp Appl Biosci 11:247-251

Seluanov A, Chen Z, Hine C, Sasahara TH, Ribeiro AA, Catania KC, Presgraves DC, Gorbunova V (2007) Telomerase activity coevolves with body mass not lifespan. Aging Cell 6:45-52

Shay JW, Wright WE (2000) Hayflick, his limit, and cellular ageing. Nat Rev Mol Cell Biol 1:72-76

Sohal RS, Ku HH, Agarwal S (1993) Biochemical correlates of longevity in two closely related rodent species. Biochem Biophys Res Commun 196:7-11
Steppan S, Adkins R, Anderson J (2004) Phylogeny and divergence-date estimates of rapid radiations in muroid rodents based on multiple nuclear genes. Syst Biol 53:533-553

Weigl R (2005) Longevity of mammals in captivity; from the living collections of the world. Schweizerbart, Stuttgart

Williams GC (1957) Pleiotropy, natural selection and the evolution of senescence. Evolution 11:398-411

Wright W, Shay J (2000) Telomere dynamics in cancer progression and prevention: fundamental differences in human and mouse telomere biology. Nature Med 6:849-851 